\title{
EDUCACIÓN SUPERIOR EN CHILE: DIVERSIDAD, DIVERSIFICACIÓN, ¿AHORA ARTICULACIÓN?
}

\section{Antecedentes ${ }^{1}$}

El Diccionario de la Real Academia Española define "diversidad" como "variedad, diferencia o abundancia de cosas distintas", "diversificación" como "transformación en múltiple y diverso de lo que era único y uniforme" y "articulación" como "enlace o unión entre dos partes de una máquina u objeto que permite y ordena su movimiento". Es decir, en tanto la diversidad se entiende con carácter estático, la diversificación y la articulación, en cambio, tienen un carácter dinámico, de proceso en ejecución con límites de tiempo no definidos.

Bien puede decirse que las instituciones de educación superior (IES) tienen la diversidad como cosa propia, siempre han sido diversas en su manera de ser y de hacerse. De acuerdo con específicas circunstancias culturales, tienden a diversificarse y/o articularse como parte importante de su manera de hacerse, constituyendo nuevas maneras de ser. La trilogía "diversidad-diversificación-articulación" acompaña desde siempre al nivel terciario de la educación, se liga transversalmente a sus otros elementos componentes y se constituye como función multivariable en la cual sus factores intervinientes cambian o se ajustan según se modifica cualquiera de los otros.

1 Las referencias principales de este artículo se encuentran en el documento elaborado por el autor en cumplimiento de las tareas encargadas al Grupo de Trabajo constituido por el Consejo Superior de Educación en el tema "Políticas Públicas", que desarrolló sus actividades entre marzo de 2005 y enero de 2006. También, en el capítulo "El Sistema de Educación Superior Chileno: La Respuesta de los 80", incluido en el libro La Educación Superior Chilena: Gestión y Administración Institucional, Foro de la Educación Superior, 1992. Llama la atención que, a pesar del tiempo transcurrido, aún están vigentes muchas de las apreciaciones allí expuestas. 
En el caso chileno, hasta fines de la década de los 70, si bien existía diversidad en la organización de los saberes intrainstitucionales, era muy escasa o nula la diversificación y casi no se requería articulación alguna. Es el marco jurídico aplicado a partir de los 80 el que posibilita un cada vez más dinámico proceso de diversificación, que sigue la tendencia general vinculada a la mayor demanda por estudios superiores, a ciertas políticas públicas y, también, a las propias estrategias de los desarrollos institucionales (Rama, 2006).

En el ayer lejano, anterior a los 80, era diversidad en la unidad (uni-versidad) y en el más cercano aparece la diversificación como elemento importante del proceso identificado como "modernización" de la educación superior. En el hoy casi futuro, diversidad y diversificación se ubican en un ambiente de educación superior inserto en un medio social en que el conocimiento es relevante. Participan en él y de él múltiples y diferentes actores y contenidos, se gestiona en mercados caracterizados de muy distintas maneras, condiciona políticas públicas ya no simplemente reactivas que deben compatibilizar y complementar las acciones estatales y privadas insertas en una modalidad de competencia (en su doble referencia de competitividad y de ser competente).

Entonces, la interrogante es si las formas y estructuras institucionales y sistémicas son consistentes con este nuevo medio social en el cual ya inciden fuertemente la internacionalización de la educación superior y las propias funciones básicas que tal medio demanda en torno al conocimiento.

La presente presión cuantitativa de más demanda, más oferta, más necesidades, más conocimiento, más investigación, más tecnología y más recursos, no puede dejar de lado ni tener preferencia sobre la presión cualitativa de mayor calidad de ejecución, mayor pertinencia, mayor equidad, mayor información, mayor innovación y mayor cooperación.

Si en educación superior se reconocen la diversidad y la diversificación como bondades, ellas requieren situarse en un 
contexto de articulación con el "hacia afuera" (el desarrollo nacional, el total del sector educación, el sector científico y tecnológico, otros centros independientes de docencia e investigación y los otros sectores de producción de bienes y de servicios) y, también, con el "hacia adentro" (coordinación de esfuerzos estatales y privados en los niveles educativos terciario y cuaternario en el logro de metas comunes).

Se refuerzan la diversidad y diversificación de la educación superior, y su consecuente articulación, por la creciente matrícula con incremento mayor que el poblacional, por el desarrollo del conocimiento, por la puesta en práctica de la educación permanente y por la existencia de opciones inter y transdisciplinarias de formación y de investigación. También, por la multiplicidad de objetivos, intereses, expectativas y experiencias educativas en la provisión de servicios que buscan responder a las necesidades nacionales, sin perder el marco de calidad, de acceso en términos de igualdad de oportunidades y, sobre todo, de respeto absoluto de la libertad académica y de la autonomía.

\section{Los hechos que son datos}

Si referimos la situación actual de las instituciones de educación superior nacionales a la tipología de diversificación postulada por Birnbaum (1983) y citada por Brunner (2006), en todas las variables que ella reconoce -la sistémica (tipos, tamaño, localización, mercado y control), la estructural (historia, estatutos y gobierno), la programática (valoración de sus capitales, cobertura de áreas del conocimiento y oferta de programas), la procedimental (tipos de servicios docentes y organización de la investigación), la reputacional (prestigio y estatus), la de clientelas (tipología de usuarios) y la de valores y clima cultural (misión y entorno social) - no caben dudas acerca de su gran diversidad y alta diversificación en amplitud y en profundidad.

En la situación nacional, buen ejemplo de lo recién mencionado, de acuerdo con la información entregada en INDICES 2007 (2006) existen 209 instituciones de educación superior en funcionamiento 
con 444 unidades operativas (166 casas centrales y 278 sedes), con un 27 \% de ellas instaladas en la Región Metropolitana. Desarrollan 7.439 carreras (6.386 de pregrado y 1.053 de postgrado y especialidades ligadas al sector salud), y atienden a 633.823 estudiantes (el 97\% de ellos en formación profesional o técnica de pregrado) involucrando a cerca de 65.000 académicos y personal docente (un 23\% con jornada completa y un $11 \%$ con jornada parcial).

La misma fuente indica que, de las carreras mencionadas -que incluyen la repetición de programas académicos entre instituciones y al interior de ellas en sedes y jornadas diferentes-, 3.783 tienen carácter profesional y 2.603 carácter técnico, y que de las 3.202 carreras que se imparten en las universidades, 673 se inscriben en Educación, 423 en Administración y Comercio, 383 en Ciencias Sociales y 179 en Derecho, entre otras áreas del conocimiento. En el caso de los posgrados, hay 692 opciones de magíster, 151 de doctorado y 210 especialidades médicas y odontológicas; del total de programas, un 41\% se localiza en la Región Metropolitana y un 13 y un 12\% en las regiones V y VIII, respectivamente.

En esta dimensión de la educación superior nacional, existe una clara superposición de las lógicas estatal y privada que, a futuro, debieran acercarse e integrarse en una tercera dimensión: la "lógica pública" que la considere como un "bien público", ya no como simple sinónimo de lo estatal, sino como la integración de esfuerzos estatales y privados, apreciados como un servicio interna y externamente articulado de modo armónico.

En este contexto, deben mantenerse la diversidad y la diversificación, buscando su fortalecimiento y consolidación en un esquema de articulación que aún no está presente en la situación nacional. Es necesario que así sea, ya que la puesta armónica de la diversidad, la diversificación y la articulación en el conjunto -y en cada una de las IES acerca el logro de la equidad a través de la desigualdad- representan una mayor pertinencia en la elaboración de los capitales culturales, su adecuada distribución geográfica y sus posibilidades nacionales e internacionales de asociación. 
Otros elementos que debemos tener en cuenta son los propios comportamientos de los sistemas de educación superior en su masificación y en la amplia diferenciación de sus relaciones internas, la búsqueda constante del aseguramiento de la calidad de servicios y productos, las demandas crecientes relativas al conocimiento, la incorporación de nuevas fuentes de financiamiento, junto con la optimización de las existentes, y la creciente incidencia de la innovación y del emprendimiento (Brunner, 2005).

Por otra parte, la introducción de regulaciones genera tensiones entre la autonomía institucional y los organismos reguladores, pues no cabe que sea el Estado el único regulador posible y menos el único agente de los mecanismos reguladores que se implementen. Existen naturales recelos respecto de que las políticas de educación superior se orienten a la construcción de una institucionalidad que posibilite tal situación, y que, con el pretexto de la búsqueda de calidad académica, se practiquen modalidades de aseguramiento y de regulación que afecten el ejercicio de la plena autonomía institucional.

En síntesis, lo que se tiene hoy en perspectiva es un modelo en el que confluyen la educación estatal y privada nacional y extranjera, cuya modalidad de desarrollo y de ejecución conlleva la aplicación de tecnologías de información y de enseñanza-aprendizaje que trascienden los límites físicos de claustros y campi.

\section{Por qué la articulación}

Ni la diversidad ni la diversificación ni la articulación de la educación superior pueden apreciarse como problemas, ya que son los medios con que se responde, efectivamente, a la creciente demanda, a las posibilidades de acceso de grupos sociales cada vez más diversos y a distintos modos de enseñar en la perspectiva de educarse a lo largo de toda la vida. La real igualdad de oportunidades requiere de una educación diferenciada, necesariamente diversa y desigual, enmarcada en un pluralismo que supone la coexistencia y convivencia de corrientes culturales diferentes. 
Si bien la articulación es necesaria, ella no puede situarse en contradicción con el criterio de autonomía, base esencial en la labor de la educación superior y punto de referencia obligado de la conveniencia de planificar, establecer y administrar mecanismos de coordinación e integración. Es el nivel institucional el principio y fin de todo el proceso, origen y destino de toda posible gestión académica y, en definitiva, unidad operativa de los mecanismos de enlace originados en las modalidades de articulación que se apliquen.

La idea de articulación también aparece como un requerimiento necesario de cumplir, en la perspectiva de la mayor potencialidad en la ejecución conjunta de tareas similares en que ese nuevo todo, de seguro, es más que la simple suma de las partes y adquiere personalidad propia. Puede constituirse como una manera de pensar que estima la educación terciaria en su integridad y significa un criterio de ordenamiento consecuente con sus elementos constitutivos, a los que reconoce como sujeto y objeto principal. No existe todavía en la educación superior chilena una lógica de articulación como servicio público -otra vez, lo "público" como integración de esfuerzos estatales y privados- y sí existen grados de descentralización en los ciclos básico y medio, que ya posibilitan pertinencias, calidades y pedagogías diferenciadas.

Su actual "des-articulación" quizás se ligue, parcialmente, a las diferencias que presentan los distintos grados de autonomía con que funcionan los tipos institucionales, con heterogéneas modalidades para su obtención, así como a las regulaciones existentes y a la incertidumbres que generan las posibles por venir -que relativizan las vigentes-, a sus muy distintos grados de aplicación y a las distintas modalidades de inserción en la libertad de mercado.

No existió "des-articulación" cuando la educación superior nacional estuvo sólo referida a un tipo institucional: las universidades, casi con carácter elitista y monopólico. Inciden en ella la concurrencia de los sectores estatal y privado, la gran diferenciación en el ejercicio de las funciones institucionales y en la calidad académica, y, también, 
la actual diferenciación institucional, universidades (Ues), institutos profesionales (IP), centros de formación técnica (CFT) e instituciones de las Fuerzas Armadas y de Orden, con distintas dependencias y canales de relación con el Estado. Asimismo, la amplitud disciplinaria (expansión y diferenciación de los saberes y de su tipología), las cada vez más amplias modalidades de trabajo presenciales y virtuales, los periodos de ciclos vitales cada vez más extensos y, ahora, la múltiple y cada vez mayor oferta de educación cuaternaria (especializaciones, postítulos, maestrías y doctorados) (Rama, 2006).

Se requieren, entonces, grados de articulación como posibilidades reales de recorridos académicos diferenciados según las capacidades e intereses de las personas, cada uno de ellos con específicas características y ya no como simples enlaces, sino como múltiples y auténticas interacciones de modalidades educativas distintas. Esto no sólo al interior de la educación superior, sino también en su relación con los otros niveles educativos y, desde luego, con todos los elementos que constituyen el desarrollo nacional, en especial con los sectores laboral, de ciencia y tecnología e inserción en el medio social, entre otros.

En la educación superior y en los otros niveles educativos, la articulación no es un fin sino un medio que permite recorridos académicos de aprendizajes personales, con pleno ejercicio de libertad de las personas en la elección y selección de sus opciones de vida. Facilita la educación permanente -hoy condicionada por la alta flexibilidad laboral existente- la reducción de la alta tasa de deserción originada, en muchos casos, por la no existencia de opciones y salidas intermedias, y la generación y sistematización de saberes inter y transdisciplinarios.

\section{La articulación en la función, forma y estructura de la educación superior}

En otros análisis realizados por el autor (Cáceres, 1992, 2005) se plantea el conocido esquema de que a la definición de "funciones" 
sigue el diseño de una adecuada "estructura", y que ésta debe adoptar una correcta "forma", integrando en este último concepto los aspectos de límites y de control.

Respecto de las funciones que debiera considerar la articulación de la educación superior, la principal y trascendente es la que corresponde al trabajo con el saber en su proyección social, en términos de su creación, ordenamiento, sistematización, transferencia y difusión, inserta en la referencia cultural, científica, tecnológica y artística que representa su medio social.

Con relación a la estructura que la articulación adopte, ella se condiciona a los elementos que inciden en su propia determinación, esto es, a las instituciones, a las personas, a las relaciones que entre ellas se generan y a los recursos materiales disponibles.

Sobre la forma de la articulación, convencionalmente ella incluye factores que pueden variar, tales como el régimen de gobierno, los procesos de creación institucional, los esquemas organizacionales que se establezcan y los modos de relación con el Estado, además de su regulación general e institucionalización.

Los avances que permitan la articulación de los actuales componentes de la educación superior nacional no pueden dejar de lado la actual confluencia de lo estatal y lo privado como asunto público de acción y responsabilidad compartidas. Existen capacidades y potencial educativo en la comunidad nacional y corresponde al Estado la principal misión de generar un régimen de libertad, en el cual cada entidad pueda asumir sus propios riesgos con plena autonomía.

En la situación vigente se postula que -por el carácter trascendente de las funciones de las instituciones de educación superior, focalizadas en el trabajo con el saber y en la gestión del conocimiento-su articulación sólo puede darse de acuerdo con las específicas voluntades institucionales y con los afanes de cooperación horizontal y vertical que ellas consideren. En cambio, sí se vislumbran requerimientos de articulaciones posibles en el conjunto de su estructura y de su forma. 


\section{La articulación de la estructura, instituciones y financiamiento}

El contexto está dado, necesariamente, por la concurrencia de otros elementos componentes, entre ellos libertad, calidad y equidad, entendidos no como valores absolutos (que no lo son), sino como propósitos que presentan grados distintos de consecución y una fuerte interrelación. Aparece, entonces, la profunda heterogeneidad de las instituciones de educación superior, en su comparación entre ellas y al interior de la misma institución, con profundas diferenciaciones en sus comportamientos.

Respecto de la libertad, buena muestra de ella es la participación de entidades estatales y privadas, en especial el significativo desarrollo de éstas como señal de la amplia aplicación del principio de libertad de enseñanza. La creación de instituciones a partir de 1981, que incluyó en el momento inicial la aparición de las "derivadas" estatales y el posterior esfuerzo cultural invertido en los nuevos proyectos, son real actualización del potencial educativo presente en la sociedad. A todas estas instituciones se les concede total independencia para la realización de sus actividades, sólo condicionada a las modalidades de licenciamiento y obtención de la plena autonomía ejercidas por el Consejo Superior de Educación (CSE), y se tiene, en lo general, un cabal ejercicio de la libertad curricular y de cátedra y de la realización de programas de investigación y extensión. Además, en términos de sus regímenes de gobierno y designación de sus autoridades, determinan sus modalidades de organización académica y administrativa según las disposiciones establecidas en sus propios esquemas jurídicos. Cabe destacar, eso sí, que el CSE cautela -y es bueno que así sea- la consistencia interna y la articulación consecuente de los múltiples y variados componentes de un determinado proyecto institucional, y que este aspecto puede constituir una adecuada base de relaciones interinstitucionales.

Son todavía incipientes los enlaces entre las entidades de educación superior pero sí se ha conformado una situación perfectible de modo 
permanente. Existen todavía pocas situaciones de cooperación y de colaboración interinstitucionales, tipo "consorcios" o "redes", en especial entre tipos institucionales distintos, y aun cuando son claras las señales entregadas por fuentes de financiamiento nacionales y extranjeras, son escasos los ejemplos en que se haya hecho realidad una fluida relación interinstitucional. Se tiene también el caso de corporaciones estatales y privadas que han creado, en su interior, la gama completa de tipos institucionales para ofrecer salida a sus egresados de otros niveles de formación y, también, la creación de instancias intermedias autónomas que complementan con opciones diversificadas a los estudiantes que no alcanzan a completar los currículos profesionales o técnicos. Asimismo, está presente aquello que "el que puede lo más puede también lo menos", con lo que se originan superposiciones en sentido descendente, afirmando la imagen social prioritaria de la universidad junto con extremas rigidizaciones internas, difíciles transferencias entre las carreras y problemáticas convalidaciones al interior de la misma institución. Hacia el exterior, priman los recelos interinstitucionales; otras veces, válidos reclamos respecto de la calidad de "los otros" cierran alternativas de interrelación y generan situaciones de difícil solución.

En los 80, incluida en la "modernización" del sector, se abre la alternativa de una primera diversificación institucional y de carreras, estratificada de acuerdo con los grados y títulos que la ley permite otorgar a Ues, IP y CFT, con delimitados campos de acción en certificaciones y validaciones de estudios. Se plantea como objetivo la permeabilidad y coordinación entre ellas, de manera de posibilitar que los estudiantes que así lo desearan y que tuvieran las aptitudes requeridas en cada nivel institucional, pudieran recorrer en términos ascendentes u horizontales las diversas opciones ofrecidas. En efecto, se señalaba como característica de este nuevo esquema que un alumno que ingresara a un CFT podía llegar a realizar estudios de doctorado en una universidad. Sin embargo, no se explicitó si tal "modernización" proponía constituir un sistema en que sus diversos componentes respondieran a objetivos comunes, lo cual requería la coordinación entre ellos como un "sistema", o si el criterio de ordenamiento surgiría de una espontánea y no regulada "coordinación sin coordinador". 
En todo caso, es posible revitalizar ahora la tesis de establecer elementos de coordinación que relacionen la tipología institucional vigente y uno de ellos puede tenerse en la articulación de los títulos, grados y validaciones que se entregan, aun cuando en esta clasificación se mezclen criterios institucionales y funcionales, teniendo en cuenta que las Ues realizan docencia de alto nivel, investigación y extensión, en tanto los IP y CFT tareas docentes de formación profesional y técnica ligada a carreras de mediana y corta duración.

Pareciera entonces que, en el futuro, en términos de la tipología institucional, lo conveniente es mantener la situación existente, esto es, tres tipos distintos (Ues, IP y CFT), pudiendo agregarse quizás un cuarto tipo institucional de libre reconocimiento (los centros académicos independientes) y enlazar de mejor manera con las instituciones de las Fuerzas Armadas a través de una similar dependencia. Obviamente, estos tipos de entidades, como definición de categorías excluyentes, se cruzan con su carácter estatal o privado y con otras variables, generándose superposiciones difíciles de apreciar y resolver. Hasta ahora, la única variable que establece este tipo de categorías es el Aporte Fiscal Directo (AFI), pues en todas las otras se tienen cruces y mezclas, dificultando los esfuerzos realizados en la búsqueda de clasificaciones coherentes.

En lo que toca a la equidad, el anterior entendimiento -sólo referido al acceso a la educación superior y a la consideración de la educación como un elemento de movilidad social relevante- se ha ampliado ahora a un concepto que la vincula también a una formación de calidad. Ya no es sólo la oportunidad de "llegar" a la educación superior, sino que ésta ha de ser cualitativamente aceptable como resguardo mínimo del buen cumplimiento de su proyección social y, como ya está dicho, para ello debe tenerse presente que la verdadera igualdad de oportunidades requiere de una educación diferenciada, necesariamente diversa.

Respecto del financiamiento, si bien es parte integral del proceso educativo e incide en su calidad de ejecución, es un componente de 
carácter accidental y, por ello, cambiante: no constituye, por sí mismo, la caracterización de una determinada articulación. A pesar de ello, sabemos que uno de los roles que el Estado debe cumplir respecto de la educación superior es el de entregar recursos para el cumplimiento adecuado de sus fines más propios. Desde la perspectiva de las políticas públicas, el diseño del financiamiento implementado a partir de los 80, modificado y complementado en varias ocasiones, puede ser nuevamente revisado en vistas de una definición más precisa de lo que se estima subsidiar por parte del Estado, de la libre concurrencia a los canales de financiamiento que se determinen y de la garantía de la igualdad de oportunidades para similares méritos académicos, todo esto en el contexto de un Estado impersonal que formula y hace cumplir normas generales e iguales para todos los individuos y sectores de la vida nacional, y que garantiza la igualdad para las instituciones y sus actores.

Entre otras, las interrogantes cuyas respuestas son las bases de futuras políticas públicas se dan, desde luego, en torno a "qué subsidiar", en términos de las funciones o tareas que representen el mayor beneficio social, ratificando lo existente o determinando nuevas modalidades de asignación de los recursos. También, a "quién subsidiar", en términos de mantener los subsidios que colaboran a la igualdad de oportunidades (créditos fiscales y becas), y a "cómo hacerlo", en cuanto asignaciones directas, incremento de los fondos concursables y apoyo a programas específicos que apunten a resolver determinados déficit. Otras interrogantes se relacionan con una reasignación distinta a la que hoy existe, en que se consideren parámetros de distribución ligados a funciones, tipos institucionales y condición jurídica de estatal y privado, o a una determinada oferta, sea ésta por áreas del conocimiento, localización geográfica o competencia (en el sentido de ser competente en la obtención de determinados resultados que interesen al país).

\section{La articulación de la forma, regulación}

Es importante la forma si se la entiende como el principio determinativo y perfectivo de una obra y con carácter accidental. 
Según esto, la articulación de las instituciones de educación superior, en cuanto a su regulación y modos de institucionalización, adopta diferentes configuraciones de acuerdo con el momento social que se tiene como referencia inmediata.

Su tratamiento corresponde a una distinta manera de analizar la intervención del Estado respecto de la educación superior. Están siempre presentes las preguntas acerca de si el diseño inicial de mecanismos e instrumentos de articulación está en su ámbito natural de acción o de si el ordenamiento y coordinación tienen que ejercerse a través de reglamentos o marcos jurídicos compulsivos. También, si caben o no intervenciones directas del Estado en las instituciones o si son efectivas alternativas como las de establecer incentivos para quienes ejecutan en buena forma sus proyectos y las correspondientes sanciones a quienes no cumplen con la oferta establecida, acompañadas de modalidades de arbitraje y evaluaciones externas y procesos de acreditación como adecuados elementos que concurren a mejorar cada entidad y, por ende, a su conjunto.

No son ni antagónicas ni excluyentes la autorregulación y la regulación externa, incluso ambas pueden darse simultáneamente de acuerdo con los niveles de trabajo en que se apliquen. Entonces, la regulación debiera entenderse como un proceso en que se expresa e integra la acción del Estado, en sus dimensiones permanentes, con las normas que las instituciones estimen aplicar, inserta en una amplia participación académica y social y con una institucionalización que otorgue importancia a los componentes regulatorios públicos, no sólo estatales. En este sentido, las labores ejercidas por el CSE, en sus procesos de licenciamiento y de acreditación, por la Comisión Nacional de Acreditación de Pregrado (CNAP) y por la Comisión Nacional de Acreditación de Postgrados (CONAP) son acercamientos a la conciliación del trabajo interno de autoevaluación con modalidades de evaluación externa ejercida por pares calificados, y establecen experiencia e información adecuadas que deben ser consideradas por la nueva Comisión de Acreditación de Chile. 
Más que hablar de privatización o de estatización de la educación superior, conceptos que suelen utilizarse con sentido peyorativo, cabe hoy día insistir en su "publicitación", en el sentido de "hacerla pública", por ser ahora terreno de lo común y de lo colectivo y, también, un bien público.

Un factor importante se liga a la información disponible sobre el balance social de lo obrado en su totalidad por cada institución: ejercicio docente, de investigación y de extensión, para el caso de las Ues, y tareas características con sus productos esperados para los IP y los CFT. Se trata de generar una base adecuada de antecedentes sobre la cual los usuarios del sistema -aspirantes, estudiantes, académicos, personal administrativo y otros interesados externos- adopten las decisiones que les corresponden en torno a opciones en la más amplia gama de asuntos. Este aspecto fundamental no tiene todavía un desarrollo suficiente en la recolección, ordenamiento y elaboración de la información pertinente para una opinión pública educada en su adecuada comprensión, y esta tarea puede ser campo de acción para organizaciones privadas, constituyendo así a la información como un natural y necesario elemento regulador.

Definir cómo avanzar desde el actual entendimiento de lo estatal y lo privado hacia un entendimiento de lo público se dificulta por los acostumbrados recelos entre lo estatal -hecho sinónimo de lo públicoy lo privado, situados en términos antagónicos y casi nunca como una posibilidad de acción conjunta. Ya no puede concebirse al Estado como único agente educador si se sabe que se actualiza el potencial educativo existente cuando se establecen las adecuadas condiciones. La tarea de la educación superior es, en definitiva, una responsabilidad compartida, facilitando cada vez más que el poder constituyente de una institución cualquiera tenga la más amplia diversidad como bien ya ganado y que nadie pretende que se pierda.

La importante intervención del sector privado en la educación terciaria no inhabilita para nada el rol que el Estado debe cumplir, relacionado con su proyección social y con el carácter subsidiario que 
le corresponde ejercer. Ligado a ese carácter, la responsabilidad estatal es promover la fe pública en torno a que la ejecución de las acciones académicas se efectúe en rangos y estándares mínimos de calidad, a la determinación de normativas flexibles y estables, y a la autorización relativa a la fundación de las nuevas entidades. Estas tareas debe cumplirlas a través de medios, instrumentos y mecanismos no discrecionales, estableciendo requisitos que compatibilicen la libertad de enseñanza con exigencias de calidad, actuando directamente o delegando esta atribución en las propias instituciones acreditadas para ello o en otro tipo de organismo autónomo. Asimismo, desde la subsidiariedad, le correspondería generar nuevas entidades cuando así lo requieran especiales condiciones de desarrollo nacional, regional o comunal, y financiar las acciones académicas propias de la educación superior, que representen el fiel cumplimiento de las funciones sociales, culturales, científicas y tecnológicas que a ésta le competen.

La determinación de mecanismos reguladores que promuevan y faciliten la articulación de las instituciones y de sus programas aparece como el ámbito natural de la participación estatal en la definición de relaciones de coordinación y colaboración interinstitucional. Ello debe posibilitar, eso sí, una alta permeabilidad entre los tipos institucionales. En todo caso, su intervención nunca podrá darse en las entidades donde sí cabe la autorregulación como propósito principal de cada una, o del grupo de éstas cuando libremente acuerden coordinarse.

\section{Referencias bibliográficas}

Brunner, José J. (2006) Diversificación y diferenciación de la educación superior en Chile en un marco internacional comparado. Disponible en http:// mt.educarchile.cl/mt/jjbrunner/archives/2006/08/diversificacion_y_ diferenciaci.html Último acceso en junio de 2007.

Brunner, José J. (2005) Tendencias recientes de la educación superior a nivel internacional: marco para la discusión sobre procesos de aseguramiento de la calidad. Disponible en http:// mt.educarchile.cl/archives/2005/08/ tendencias_reci_1.html Último acceso en junio de 2007. 
Cáceres, Eugenio (1992) El Sistema de Educación Superior Chileno: La Respuesta de los 80 En: La Educación Superior Chilena: Gestión y Administración Institucional. Santiago de Chile: Colección Foro de la Educación Superior, CEP-CPU-FLACSO.

Cáceres, Eugenio (2005) La Diversificación en el Sistema de Educación Superior Chileno. ¿Existencia o Supervivencia? Documento interno del Grupo de Trabajo "Políticas Públicas" del Consejo Superior de Educación.

Cáceres, Eugenio (1988) Bases para el diseño del Sistema de Educación Superior en Chile. En: La Educación Superior Privada en Chile. Antecedentes y Perspectivas. Santiago de Chile: Corporación de Promoción Universitaria (CPU).

Ministerio del Interior (1981) Declaración sobre la nueva legislación universitaria.

Rama, Claudio (2005) La Tercera Reforma de la Educación Superior en América Latina. Disponible en www.udlap.mx/rsu/pdf/3. Último acceso en junio de 2007.

Rama, Claudio (2006) Las Reformas de la Educación Superior en América Latina en el contexto de la sociedad del conocimiento. Disponible en http://www. slideshare.net/claudiorama. Último acceso en junio de 2007.

Recibido: 23 de abril de 2007

Aceptado: 11 de junio de 2007 